\title{
RIPK1 protects epithelial cells
}

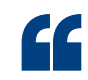

\section{the scaffolding \\ function of}

RIPK1 has a

role in vivo

in regulating

epithelial cell

survival and

maintaining

tissue

homeostasis

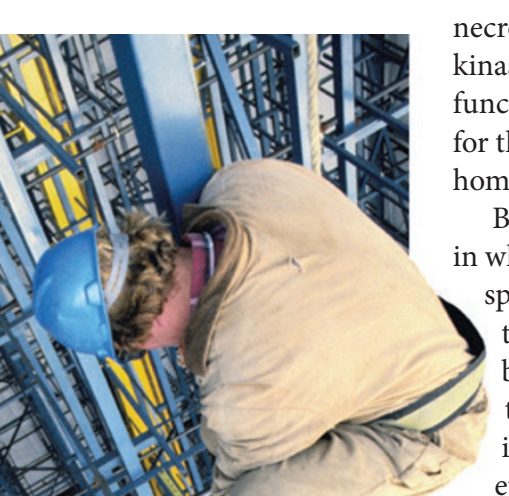

Receptor-interacting protein kinase 1 (RIPK1) is a Ser/Thr kinase that has a key role in cell death and inflammation initiated by death receptors. RIPK1 can promote two cellular fates downstream of TNF receptor 1 (TNFR1): NF- $\kappa B-$ mediated cell survival, and caspase 8 (CASP8)-mediated apoptosis or RIPK3-dependent necroptosis (also known as regulated necrosis). The in vivo role of RIPK1 has been difficult to ascertain because RIPK1 knockout mice die perinatally. Now in Nature, two studies report that RIPK1 can inhibit intestinal epithelial cell apoptosis and keratinocyte necroptosis in mice through a kinase-independent scaffolding function, and that this is important for the maintenance of tissue homeostasis.

Both groups generated mice in which RIPK1 was knocked out specifically in the intestinal epithelium. These mice had reduced body weight and died prematurely; RIPK1 knockout caused intestinal inflammation and epithelial cell apoptosis resulting in villus atrophy and loss of different cell types including Paneth cells, goblet cells and enterocytes, leading to physical barrier dysfunction and intestinal leakage.

To understand how RIPK1 maintains intestinal epithelium integrity, both groups analysed the effects of RIPK1 knockout in the absence of TNFR1. Ablation of the $t n f r 1$ gene reduced apoptosis and ameliorated the intestinal pathology, protecting mice from death. Thus, although the intestinal pathology in RIPK1knockout mice seems to be mainly induced by TNFR1-dependent signalling, TNFR1-independent pathways also contribute to it.
Next, the authors tested whether blocking cell death in intestinal epithelial cells would rescue the RIPK1 knockout phenotype. Both groups observed that deleting Ripk3 did not have any effect, suggesting that RIPK3-dependent necroptosis is not the primary pathway that induces intestinal cell death in these mice. Takahashi et al. also found that deleting Casp 8 completely protected the intestine from apoptosis and inflammation, identifying RIPK1 as a suppressor of CASP8-dependent apoptosis. Moreover, they report that treatment with antibiotics as well as crossing with animals lacking a major adaptor of the Toll-like receptor system significantly prolonged survival, suggesting a contribution of commensal bacteria in the pathology.

Dannappel et al. observed that epithelial deficiency of FASassociated death domain (FADD; a death receptor adaptor) strongly reduced apoptosis and wasting in RIPK1-knockout mice, but resulted in caspase-independent death of epithelial cells and in altered intestinal pathology, which were rescued if RIPK3 was also depleted. Thus, RIPK3 induced RIPK1independent necroptosis in FADDdeficient epithelial cells. This was surprising, as RIPK1 is known to be essential for RIPK3-mediated necroptosis.

As RIPK1 has both kinasedependent and -independent functions, both groups analysed knock-in mice expressing a kinasedead RIPK1. The mice showed no signs of inflammation or apoptosis, which indicates that these were caused by a loss of a RIPK1 scaffolding function rather than its kinase activity. Dannappel et al. also found that TNF stimulation induced the degradation of pro-survival proteins in RIPK1-deficient cells, but not in cells that expressed kinase-dead RIPK1. This suggests that the scaffolding function of RIPK1 prevents the degradation of these proteins when recruited to the TNFR1 signalling complex. Interestingly, Takahashi et al. reported that in cultured organoids the levels of NF- $\kappa \mathrm{B}$ signalling remained intact by RIPK1 deficiency, indicating that the role of RIPK1 in promoting cell survival is independent of NF- $\mathrm{BB}$ signalling. Moreover, Dannappel et al. observed that keratinocyte-specific RIPK1 deficiency, but not lack of its kinase activity, triggered RIPK3-dependent necroptosis and severe skin inflammation.

Together these studies show that the scaffolding function of RIPK1 has a role in vivo in regulating epithelial cell survival and maintaining tissue homeostasis. RIPK1 prevents inflammation in the skin by inhibiting keratinocyte necroptosis, and preserves vital intestinal functions by inhibiting intestinal epithelial cell apoptosis. The mechanisms that lead cells to be more prone to either apoptosis or necroptosis in the absence of RIPK1 remain to be elucidated, but likely do not involve NF- $\kappa \mathrm{B}$ signalling.

Kim Baumann

ORIGINAL RESEARCH PAPERS Dannappel, M. et al. RIPK1 maintains epithelial homeostasis by inhibiting apoptosis and necroptosis.

Nature http://dx.doi.org/10.1038/nature13608 (2014) | Takahashi N. et al. RIPK1 ensures intestinal homeostasis by protecting the epithelium against apoptosis. Nature http://dx.doi.org/10.1038/ nature13706 (2014)

FURTHER READING Yuan, J. \& Ofengeim, D. Regulation of RIP1 kinase signalling at the crossroads of inflammation and cell death. Nature Rev. Mol. Cell Biol. 14, 727-736 (2013) | Vanden Berghe, T. et al. Regulated necrosis: the expanding network of non-apoptotic cell death pathways. Nature Rev. Mol. Cell Biol. 15, 135-147 (2014) 